\title{
生物物理学的現况及对其在我國發展的意見
}

\author{
婁 成 后
}

\author{
(北京费業大学 中國科学院植物生理研究所)
}

生物物理学是近些年來才独椡一蚡的一門生物科学, 虽然在百余年前已經有人开始 研究有机体的电学現像, 然而它的迅速發展却是较近的事, 是在生物学、物理学与化学累 皘了一定的知識以后才实現的。生物物理学的任务大致可以分为三方面:

一、研觉有机体生活环穓中物理因素与生物的关系及其对生物的影响，像幅射、温 度等重要的物理因素如何参加生物的新陈代謝及如何改变它; 在不同情况下, 如何設計

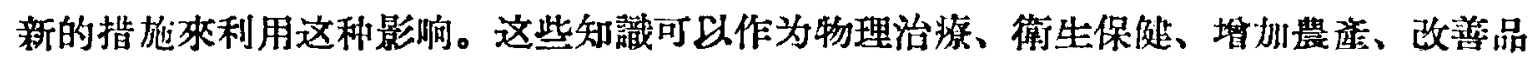
蚠与誘導变异的依据。

二、研究生物体中物理化学的構成与其变化, 深入地探討与生物形态轉变密切关联 的能量㬍变和物質轉变, 在实踐中可以更好地控制与利用这些过程与結粠。

三、利用物理学中的精密仪器与方法來探測生物与其环境的动态, 以及有机体闪的 生理变化。精致的物理仪器最椬長于在不大摸摄对象的正常活动中, 探测它的極共細微 与瞬息的变化。这种知墥可以作为医学与農業上物理診断与生理指标的根据。

- 現在就生物物理学中已經發展的几个部門分別討論一下。

\section{一 电生理学}

生物物理学中歷史最悠久与最發达的一个部門是电生理学, 可以證, 它与电刘力学 同一淵源。伽伐尼用雨种金屬刺激青蛙腿的有名試驗, 一方面是电生理学的开端，另一 方面促成了弗打电池的發明。有了这样的稳恒电流的來源才導致电磁学与电解算化学的 發展, 而后雨者在理諭与方法上的成就反过來及大大地促進了电生理学的進步。現代生 物物理学对电学知識的要求是多方面的，几乎每一項研究都缡不开电学設备。

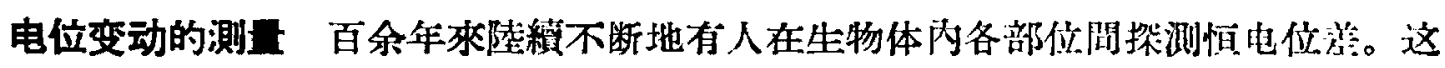
种电位差顯然随有机体的生理狀态而改变, 生物一旦受伤或死亡, 它的穞恒电位也就念 剧下降。但是由于生活組皒中分流途徑的难于控制，这种测量不易作到精确。对于其与

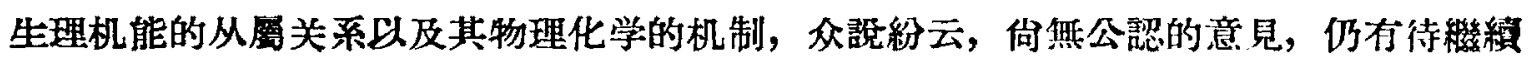
研究。近來在电位測量仪器上与㙷微操作上有了改淮, 已䋔可以作到测量一个緗胞內外

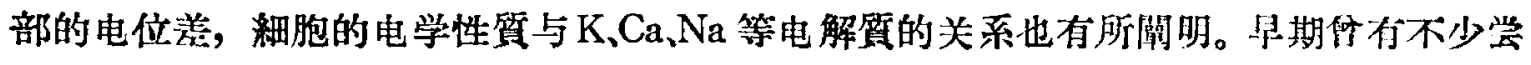

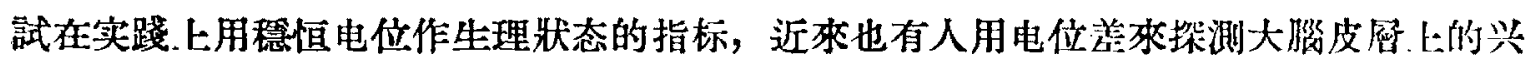
奋区，以及來判断植物的抗塞性等。但总的證來由于上述的困难, 向未作到确切有用的 地步。 
多一方面，在生物体內变动迅速与周期性的电位变化的研究收穫较大。动作电位的 测量, 是研焭生物体內刺激的傅遁, 以及研究神經肌肉与威覚器官的生理, 最得力而不可 少的。在动作电波的細節及其与穞恒电位、电阻抗等的关系上最近也有智著的成精。当刺 激强度增加时, 局部电反应随着加强，到达一定程度一轉而爆發出不連䊀的波动。但欧 美有些生物物理学家对神經的兴奋与抑制过程的分析, 然旋在数学方程式的描遮上, 对 其本筫与机制的榊明上，还没有得到長足的進步。植物中的动作电位，僅有少数的研究，

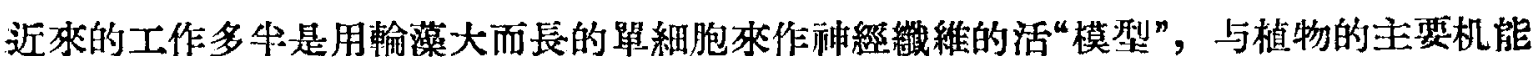
沒有發生联系。

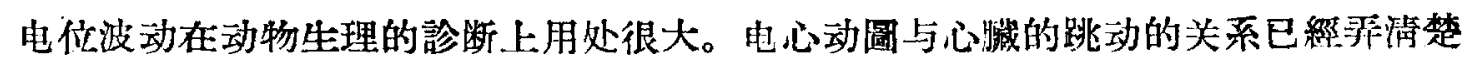
到可以用來作䠛床彭断, 这已为人所習知。电腦动圆地与大腦皮曆的活动（如視敩）建 立了一些联系, 将來在商級神緸活动的研究上会有大的發展。

电阻抗的改变 生物的电阻抗也随其生理狀态而改变。本世紀初已有些試驗建立海 澡的电阻抗与原生丝透性的关系。生物高退与死亡时，电阻抗也急剧下降。稍后的試驗 部时当动植物細胞受到刺激而引起兴奋, 在动作电位出現的同时, 电阻抗也有相应的臨 时降落、鉀离子的问外釋放等，用对各种頻率交流的阻抗或对电脉冲的睡息反应可以测 出。随㨁流电刺激的方淌不同, 細胞的电阻抗, 与其电位反应一样, 也有不同, 換们話 説，有整流作用。生物的电位与电阻抗容易受外界条件影咃而改变，在兴奋时的臨时降 涪, 以及电阻抗的整流性筫等, 都使人联想到半導体的性啠。

生物电阻抗与整流作用可以作为生理效应的指标, 在实际上也管你人作过这利尝試,

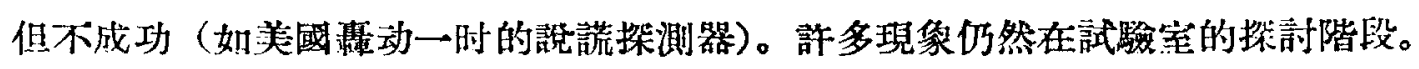

各种电流的效应生物中刺激与反应的关系, 电刺激作得最为完备。利用电学設备,

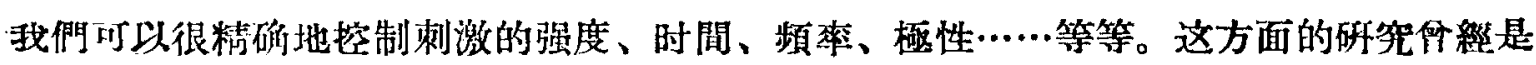
20 世紀初期电生理学研究的中心問題之一, 妌从而發現了刺激的时間因菜的重要, 以及 探討了在不同条件下生物反应中兴奋与抑制过程的相互关系。这些資料很可供其他物理 化学刺激对生物效应的参考。

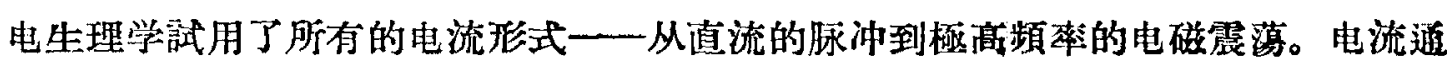
过有机体时件随着一些物理化学的过程, 秚且引起極其复雜的生理反应。每种电流都有 它的特殊效应: 如直流的电解与極性效应, 滈頻率电流的透热效应, 超声波的引起細胞 結構的解体等等; 不问組織在不同情形下对之發生各种反应，如正或負的趋性与向性反 应，兴奋或抑制的反应等，随处理的剂量与生理狀态而定，大有研究的余地。

正是因为我們研究电流的各种生物效应比較周到，在医燎与農業实践，上也找到了多 样的应用。㨁流电的刺激、磁醉、电解、电泳、趋电性等效应已用在医潦与㵂業上。滈

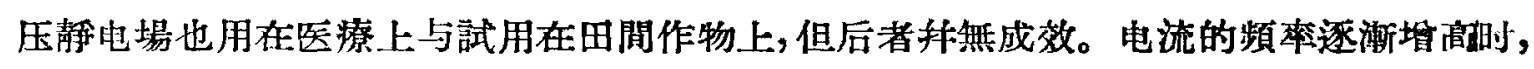
对組織的刺激效应减弱, 而加热的效应剒强。滈頻䇣的透热作用对不同組織可以分別的 由內部加热, 不需要直接的接触, 在医療上用处很多。近來的試駗高頻策电流可以与基 空等用來于燥和子等農落。也可以用在种子播种前处理, 刺激早期生長与用來作巴氐 瑊菌等。超声波振落在低剂量时也有刺激生長的效应, 較强剂量引起細胞粘構的破坏, 可用來減藮, 在農差品加工上可用來促淮乳化。

6 月号

科学通报 


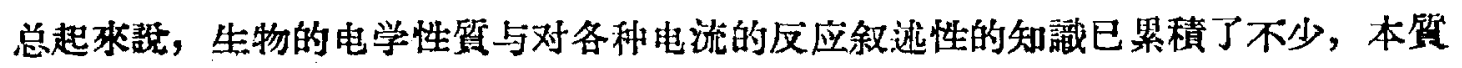
的探討还在初期。电翮断、电处理与治療, 在医学上已狙得席泛的应用, 在農業上还只 是开端。

\section{二楬射生物学}

太陽光譜 太陽光中的可見部分的生理效应在动植物学里都有光耀的开端。这就是 亥姆霍茹在䙹喾生理上与季米里亞捷夫在光合作用上的工作。現在这兩方面的研究都引! 入了光量子的理論。綵叶利用陽光造食物是一切生命活动所仰借的, 生物物理学家㳩近 皘極参加了光合作用的研究。光的生理效应不僅如此，光照的久㟻与光畹的交替（光周 期)、光諳的組成和强度, 对生物中的化学过程、發有的進度、器官的形成, 以及机休的

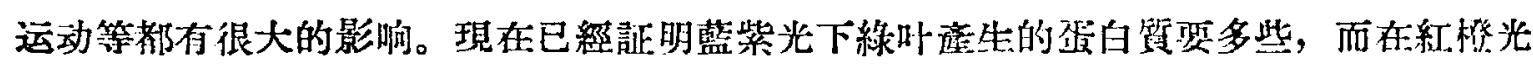
下則更富于糖类。在人工光照下载培植物与飼养动物、新光源的設訐、在筫与星上准确 地控制以及生物充分利用光能的綜合措施等等, 也在生物物理学研究机構研馀一列。太 昜光的不可見部分, 紅外馢的热輻射, 已用在医療与生物化学分析中。紫外桡也击在动

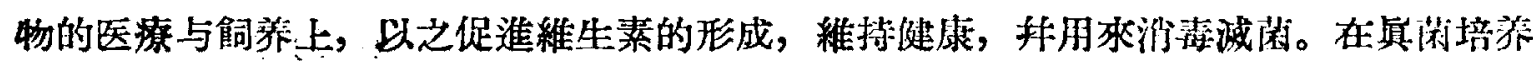
中利用紫外馢來誘菜变异。

生物組䅧不僅受光的影响，在活动时也放出射綫來，有的是可兄光（如签火出），存

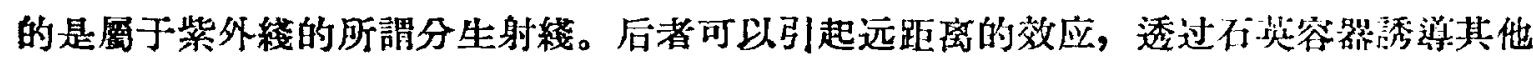

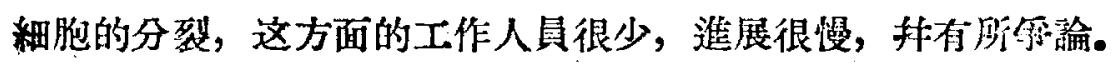

$\mathrm{X}$ 射結与同位素的放射性 原子能被人类發現与利用以后，輻射生物学饾一个最游

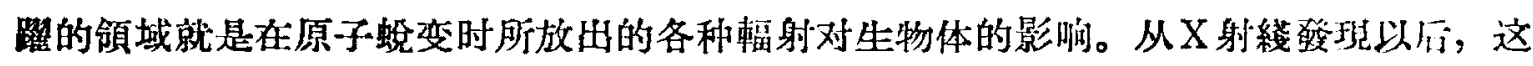

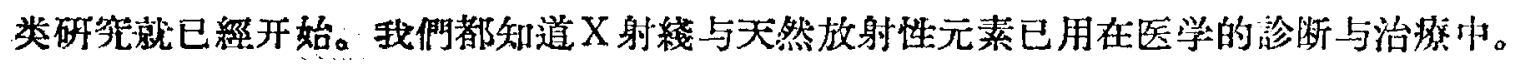

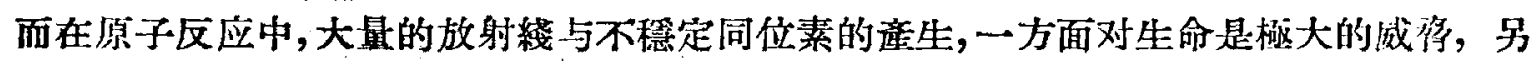

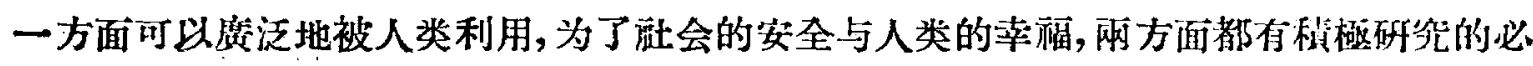
要。各种射綫一中子流与各种“硬度”的 $\alpha, \beta, \gamma$ 射綫一一对生物可以引起多力两的影响, 其物理化学的机制正在研究之中，时常被認为是通过射綫在体队水相中门起的电琝作州。

放射捗同位素在生物学研究上主要有兩条途徑可循:

第一，同位素的放射性和X射綫一样，有刺激与抑制活組䄉的生長与發有的作用， 能够誘算出遺傅性上有利的变异，以及消除生物体与其制品中的病虫整。随着放射棧剂 量的增加与租織的生理狀态的不同，对生物可以引起相反的效应。極低剂呈的身綫则激 有机体的生長与發有, 可以用在增進田間作物的憧量上; 誘導遗傅性的变异, 可以用在 改良作物的品筫上。略高的剂量就反而抑制組織的生長与其他活动, 可以用在滛病的治

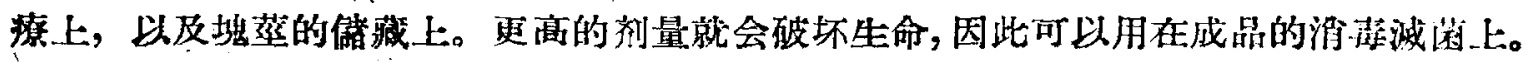
放射綫对生命活动随剂量与組織的不同而引起刺激、抑制甚或表害的效应，要結合有机 体对刺激反应的一般規律加以研究。

第二，放射性的同位素虽然剂量極小，在变化移动之中随时随刻可以斯示出自己的

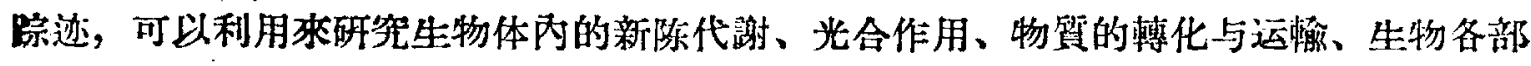
位之間、生物与外界之間的相互关系; 可以利用作医療上的钐断, 以及供給農業实践的 
科学根据。

\section{三 热与力能学及其他}

如果說研究生物体內物筫的䡯变, 我們要借重生物化学, 則在研究能量的轉变上, 就恐沿重生物物理学。在热量的釋放与食物的湓耗上的研究，首先把一般物質运动中的 能量守恒定律在生活物筫中建直起來，巩固了生物学的唯物覌点。生理研觉中已然应用

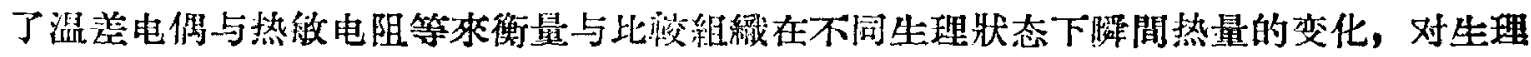

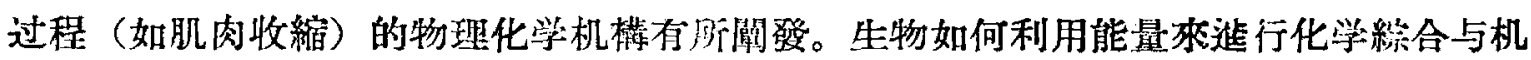
械工作等虽已有了端倪，但許多地方还是待解之謎。

温度的迅速或㸞期性的改变对生物有多方面的影响，諸如刺激作用、促進与抑制新 陈代侧、發有的進程、物質的分配、器官的形成等等。有些效应已用在医澄（如各利热 療法）与農業中（如利子处理）。

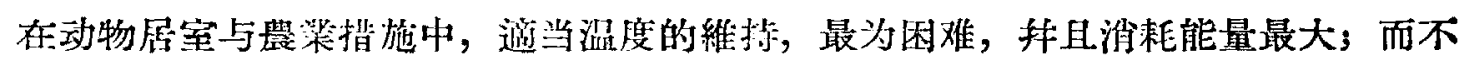
同的生理过程只有慰格地在一定温度范四入才能進行。从北京的冬季温宝与陽畔伡培与 夺葫的經驗可以看出, 能够維持適当的温度, 就可以大大地延長生長季節与复利面皘。 因之对地球上热源的光分利用，如太陽輻射热、地心热等，土㩁的保温与導热性能与裝

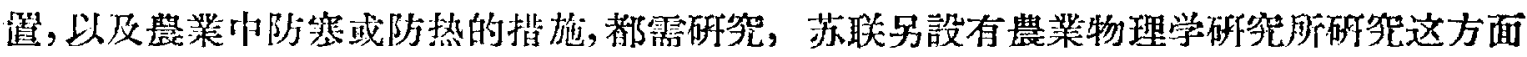

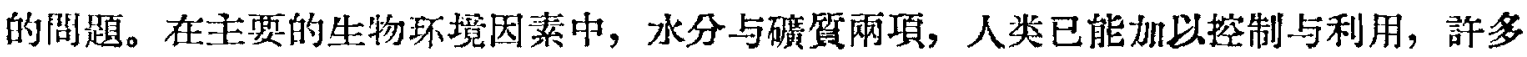

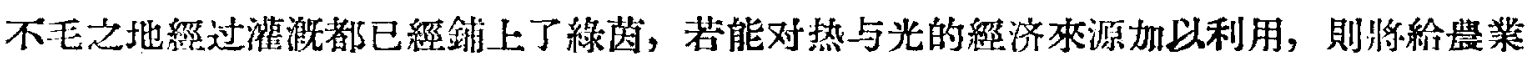

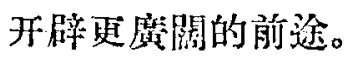

其他如在滴空、樑海、滈速度的航行中，所發生的压力与重力的剧变对生物所發生 影响的呼究，在征服大自然与軍事防御中占重要地位。这类研究在第二次圮多大战中， 由于日新月异髚速嵪压的新武器的出現, 淮展得比較迅速。在和平共处的世界里, 高空

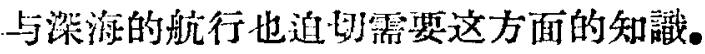

\section{四 超顯徽結構及精密仪器的使用}

为了樑入研究生活物質及其行生物結棰的特胙、形成过程、与在执行机能时內部的

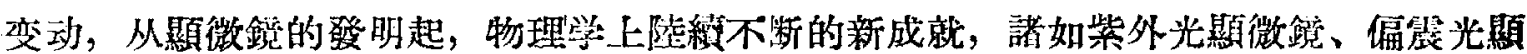

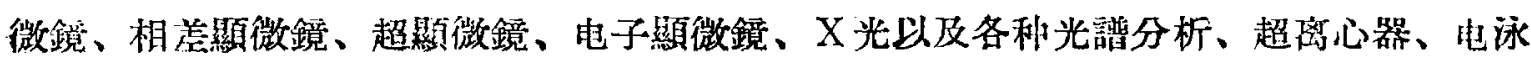

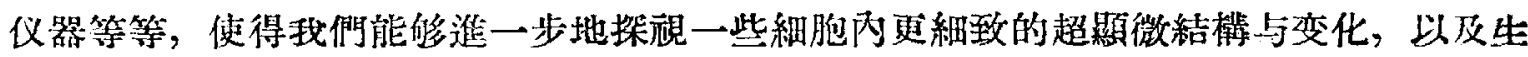

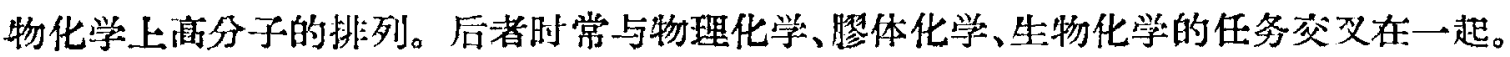

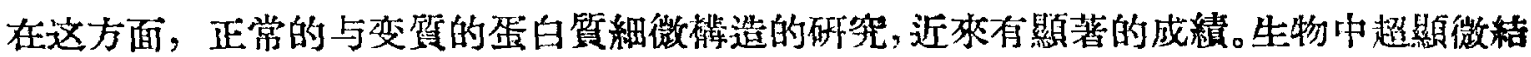

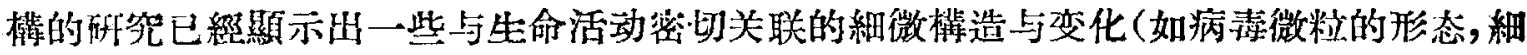

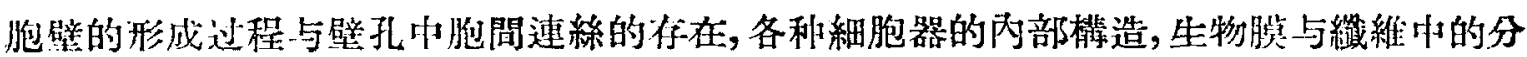
子排列, 肌肉收維时內部排列的变化等等), 这些工作不論存理論，上还是在实踐 上都有很 大的意义。在原生貿的結權中，局部的些微影响往往会引起全部原生買結構的反应，在 生物的細微的結桠的中問有“远程力量”在起作用的問題引起了一些人的注意。超㙷微結 搆的研究在自然的与人造的緎維的工業利用上都有不少的帮助, 早期对生物形态机极力 
学上的研究由此又推進了一步。这种研究往彺要配合着有关部門共同來淮行。在精復物 理仪器的設計与使用上, 生物物理学家有責任來貢献出自己的等長协助其他生物学者共 同解决一些問題。

\section{五 我國的現况及应有的准备}

生物物理学是一个边緣科学, 与許多接近的科学如細胞学、生理学、生物化学、物

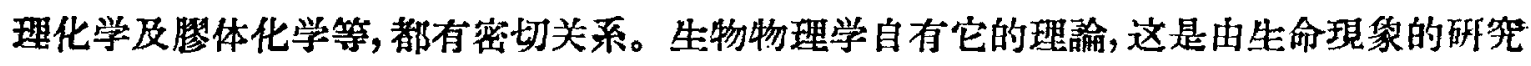
上發展出來的。因之有机界中的物理过程不能与它的生物学背景脱缡, 正像土垻的物理

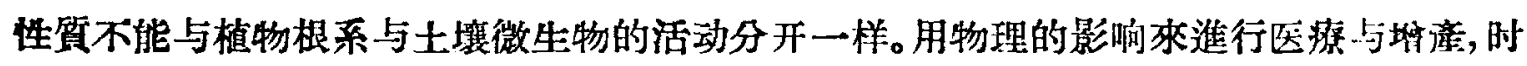
常是一种豧助手段。生物物理学涉及的䦎題中，也有是应用物理学上的新方法（如示踪 原子) 來解决生物学上的問題。若对原專業問題沒有清楚的認識与明确的目标, 期使有 这样的新工具也繁济于事。

然而另一方面, 物理学的知識对这門科学却非常重要。生物物理学的問題只有对物 理学有修养的人才能提得出，才能深入地研討。从以上儿節那述可以看到电生理学对电 磁学与电解質的物理化学的需要。从电子管綫路發展出來的計算机与控制論, 無疑地将

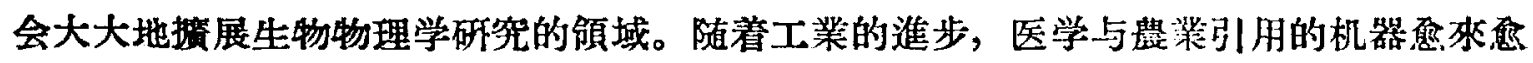

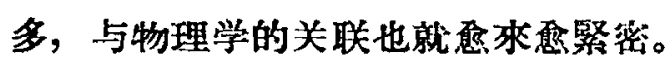

我國在生物物理学的研究中, 工作比较多的是在电生理学方面, 然而就現狀來看, 为数还嫌过少。其他方面如射綫与电流医療, 紫外綫、滈頻本电流与 X射綫对犯子与种 子的处理, 示踪原子在生物体內的变动, 虽然也有些工作, 多生挶重在实际应用上, 很

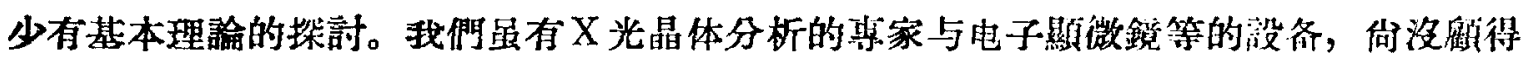
及与生物的結粠發生联系。在改善生物的生活环境中, 呼究地面上热量的保持与利用,

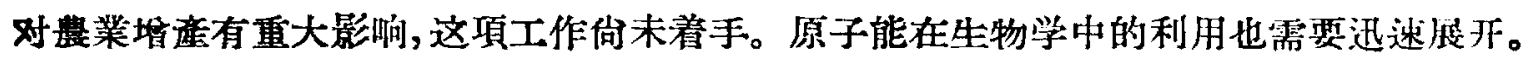

照現有情况桃看, 开展生物物理学的工作自然是訓練人才培养干部为首繁任务。这

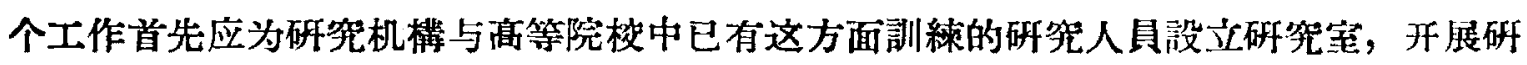

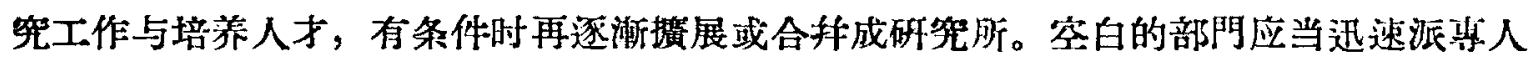
到有关部門学㚙或出國留学。在綜合性大学生物系应該考慮添設生物物理学的誤程及尃

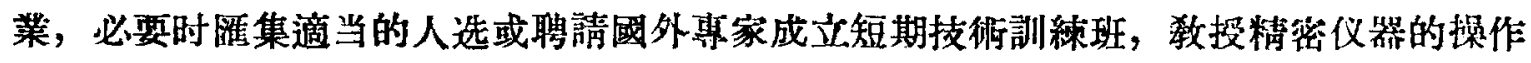
和示踪原子的使用等。農業和医学的專門研究机桡, 要及时地应用物理学上的新仪器和 新方法，协助解决専業問題。 